\title{
Emulation-based semiglobal output regulation of minimum phase nonlinear systems with sampled measurements
}

\author{
Daniele Astolfi ${ }^{1}$, Giacomo Casadei $^{2}$ and Romain Postoyan ${ }^{1}$
}

\begin{abstract}
We investigate the semiglobal output regulation of minimum-phase single-input single-output nonlinear systems with sampled measurements. We proceed by emulation. We start by considering a continuous-time regulator, which solves the problem in the absence of sampling. Then, we consider sampled measurements and we model the overall system as a hybrid system. We show that the original continuous-case properties are preserved when the measurements are sampled provided that the maximum allowable transmission interval satisfies a given explicit bound.
\end{abstract}

Index Terms-Output regulation, nonlinear systems, networked control systems, sampled-data.

\section{INTRODUCTION}

Networked control systems (NCS) have become a popular research topic in the last few years. Not surprisingly though, since in modern applications, controllers and plants often communicate over a multi-purpose network, which may be shared with other tasks. It is a well known fact that, in contrast with point-to-point connections and dedicated hardwares, sharing the communication channel may have a major impact in making a control system more flexible, in reducing the implementation cost and in simplifying the maintenance. However, the communication channel induces uncertain transmission intervals, network delays and packet loss in general, see e.g. [1], [2], which may destroy the desired control properties, if ignored. There is therefore a need for adapted control design methodologies, which cope with these constraints.

The vast majority of the literature on NCS focuses on the stabilization problem, see e.g. [3]-[6], or on the observer design problem, see e.g. [7], [8]. Only recently, some researchers have addressed the problem of output tracking, e.g. [9]-[11], where it is shown that the design and the analysis exhibit specific problems, which are mainly due to the feedforward action of the controller. Very few works, on the other hand, have investigated the output regulation problem for NCS, namely the scenario in which we want to regulate some desired outputs to a given reference while rejecting other external disturbances. In this framework, output tracking and disturbance rejection are usually addressed concurrently, resulting in a problem which is more challenging and harder to address with respect to the purely output tracking scenario.

\footnotetext{
1 Université de Lorraine, CNRS, CRAN, F-54000 Nancy, France \{daniele.astolfi@univ-lorraine.fr, romain.postoyaneuniv-lorraine. fr .

2 Univ. Grenoble Alpes, CNRS, Inria, GIPSA-lab, France, \{giacomo.casadielgipsa-lab.fr\}.

This project has received funding from the "Région Grand-Est" and from the European Research Council (ERC) under the European Union's Horizon 2020 research and innovation programme (grant agreement $\mathrm{N}^{\circ} 694209$ ).
}

While output regulation has been solved for continuoustime linear systems by Francis and Wonham with the celebrated internal model principle [12], this problem is still largely open for continuous-time nonlinear systems in general, though remarkable results have been obtained for minimum-phase nonlinear systems in a semiglobal context (namely for any arbitrarily large compact set of initial conditions), see e.g. [13]-[17] and in cooperative network contexts [18]. The scenario where the measurement and the control input are sampled has been studied mainly for linear systems assuming periodic sampling. One of the main focus in this direction was the development of control strategies able to avoid ripples between inter-sampling times, see for instance [19]-[21]. A first attempt to address nonlinear systems can be found in [22], where the authors consider sampled and quantized measurements, but bounds on the output regulation error and the maximum allowable transmission interval (MATI) are not explicitly computed. Recently, the nonlinear output regulation problem has been studied in [23], where the authors propose an event-triggered law for the case of sampled inputs and sampled outputs achieving practical output regulation.

In this work, we consider the scenario where the plant is nonlinear, single-input single-output, minimum phase and its output is sampled, not necessarily periodically. We first design the controller ignoring the sampling by following [15]. It appears that we cannot apply the results in [15] "off the shelf", as we need bounds on the controller parameters, ensuring output regulation to compute the MATI afterwards, bounds which are not given in [15]. We therefore slightly strengthen the assumptions in [15], and revisit the analysis to obtain the desired bounds, using linear matrix inequalities (LMI). We then take into account sampling and we model the overall system as a hybrid system in the formalism of [24]. We prove that output regulation is solved provided the gains of the controller are sufficiently large, like in the continuous-time case [15], and the MATI satisfies a given explicit bound. The approach we take is inspired by [6], where the stabilization of the origin problem for nonlinear sampled-data systems is investigated. While, the model and the proof techniques we present are similar to those in [6], the output regulation problem has its features, which makes the result of [6] not directly applicable. The results are then illustrated by a nonlinear example for which it appears that the MATI estimates are reasonably tight.

The paper is organized as follows. In Section II, the required results on the continuous-time problem are provided. In Section III, we present the hybrid system 
model of the continuous-time nonlinear system with sampled measurements and we provide the main contribution of this paper. Finally, a numerical example is given in Section V.

Notations and definitions. $\mathbb{R}$ denotes the set of real numbers, $\mathbb{Z}$ the set of integers, $\mathbb{Z}_{>0}:=\{1,2, \ldots\}$ and $\mathbb{Z}_{\geq 0}:=$ $\{0,1,2, \ldots\}$. For $x \in \mathbb{R}^{n},|x|$ denotes the standard Euclidean norm, while $|x|_{\mathcal{A}}:=\inf _{a \in \mathcal{A}}|x-a|$ denotes the distance from $x$ to a set $\mathcal{A} \subseteq \mathbb{R}^{n}$. Given an open set $\mathcal{B} \subseteq \mathbb{R}^{n}$ containing a compact set $\mathcal{A}$, a function $\omega: \mathcal{B} \rightarrow \mathbb{R}_{\geq 0}$ is a proper indicator for $\mathcal{A}$ on $\mathcal{B}$ if $\omega$ is continuous, $\omega(x)=0$ if and only if $x \in \mathcal{A}$, and $\omega\left(x_{i}\right) \rightarrow \infty$ when $x_{i}$ approaches the boundary of $\mathcal{B}$. We define (see [17, Appendix A])

$$
|x|_{\mathcal{A} / \mathcal{B}}:=\left(1+\frac{1}{|x|_{\partial \overline{\mathcal{B}}}}\right)|x|_{\mathcal{A}}
$$

for any $x \in \mathcal{B}$, where $\partial$ denotes the boundary of a set and $\overline{\mathcal{B}}$ denotes the closure of $\mathcal{B}$. The function $|\cdot|_{\mathcal{A} / \mathcal{B}}$ is a proper indicator for $\mathcal{A}$ on $\mathcal{B}$. Given two functions $f, h$, we denote with $L_{f} h(x)$ the Lie derivative of $h$ along $f$, namely $L_{f} h:=\langle\nabla h(x), f(x)\rangle$ where, given two vectors $a, b \in \mathbb{R}^{n},\langle a, b\rangle:=a^{T} b$, and given a scalar field $h$, we define $\nabla h(x):=\partial h(x) / \partial x$. Throughout the paper, the matrices $(A, B, C)$ are in prime form of suited dimension, namely

$$
\begin{aligned}
& A=\left(\begin{array}{cc}
0_{(n-1) \times 1} & I_{n-1} \\
0 & 0_{1 \times(n-1)}
\end{array}\right), \quad B=\left(\begin{array}{c}
0_{(n-1) \times 1} \\
1
\end{array}\right), \\
& C=\left(\begin{array}{ll}
1 & 0_{1 \times(n-1)}
\end{array}\right),
\end{aligned}
$$

where $n \in \mathbb{Z}_{>0}, I_{n}$ denotes the identity matrix of dimension $n$, and $0_{n \times m}$ denotes a zero matrix of dimension $n \times m$. The symbol $\star$ stands for the symmetric entries in a matrix.

In this work, we consider hybrid systems of the form [24]

$$
\dot{x}=F(x), \quad x \in \mathcal{C}, \quad x^{+}=G(x), \quad x \in \mathcal{D},
$$

where $x \in \mathbb{R}^{n_{x}}$ is the state, $\mathcal{C}$ is the flow set, $F$ is the flow map, $\mathcal{D}$ is the jump set and $G$ is the jump map. We assume that the hybrid model satisfies the basic regularity conditions, see Section 6.2 in [24], which will be the case in our study. We recall some definitions from [24]. Solutions to system (1) are defined on so-called hybrid time domains. A set $E \subset \mathbb{R}_{>0} \times \mathbb{N}$ is a compact hybrid time domain if $E=$ $\bigcup_{j=0}^{J-1}\left(\left[t_{j}, t_{j+1}\right], j\right)$ for some finite sequence of times $0=$ $t_{0} \leq t_{1} \leq \ldots \leq t_{J}$ and it is a hybrid time domain if for all $(T, J) \in E, E \cap([0, T] \times\{0,1, \ldots, J\})$ is a compact hybrid time domain. Given two hybrid times $\left(t_{0}, j_{0}\right),\left(t_{1}, j_{1}\right) \in E$, we denote $\left(t_{0}, j_{0}\right) \leq\left(t_{1}, j_{1}\right)$ if $t_{0} \leq t_{1}$ and $j_{0} \leq j_{1}$. Given an initial condition $x^{\circ} \in \mathcal{C} \cup \mathcal{D}$, we denote by $x(t, j)$ a solution to the hybrid system starting at $x^{\circ}$ at time $(t, j)$ if $(t, j) \in$ $\operatorname{dom} x$. Throughout the text, we will refer to solutions as maximal solutions, see Definition 2.7 in [24].

\section{Problem Statement}

Consider the system

$$
\begin{aligned}
& \dot{z}=f(w, z, x) \\
& \dot{y}=q(w, z, y)+u
\end{aligned}
$$

where $(z, y) \in \mathbb{R}^{n} \times \mathbb{R}$ is the state, $y \in \mathbb{R}$ is the measured output to be regulated to zero and $u \in \mathbb{R}$ is the control input ${ }^{1}$. We suppose the initial conditions of the $(z, y)$-system range in some arbitrarily large given compact set $Z \times Y \subset \mathbb{R}^{n} \times \mathbb{R}$. The bounded exogenous input $w \in W \subset \mathbb{R}^{r}$, represents a disturbance to be rejected or a reference to be tracked and it is supposed to be generated by an exosystem which is Poisson stable ${ }^{2}$ of the form

$$
\dot{w}=s(w) .
$$

Finally, the functions $f, q, s$ are smooth enough.

In this work we consider the scenario depicted in Figure 1 in which a network is used to connect the sensor to the regulator. We focus on the effect of sampling, and ignore delays and quantization. Transmission over the networks occur at times times $t_{i}, i \in \mathbb{Z}_{\geq 0}$, satisfying

$$
0<v \leq t_{i+1}-t_{i} \leq T
$$

where $T$ is the MATI and $v$ is the lower bound on the minimum achievable transmission interval given by the hardware constraints. The inter-transmission times $t_{i+1}-t_{i}$ may be time-varying and uncertain. In the following, the sampled version of $y$ is denoted as $\hat{y}$ and it corresponds to the most recently transmitted output value. The case where $u$ is sampled will be addressed in future works as it leads to some non-trivial technicalities, similar to those encountered in the tracking control of NCS [11].

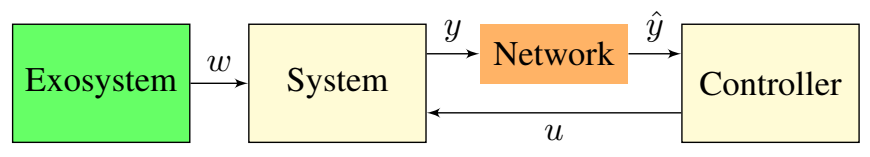

Fig. 1. Output regulation with sampled measurements.

In this context, the problem of output regulation under sampling measurements amounts to design a controller of the form

$$
\dot{\xi}=\varrho(\xi, \hat{y}), \quad u=k(\xi, \hat{y}),
$$

with initial conditions ranging in a compact set $\Xi$, such that the trajectories of the closed-loop system originating from $W \times Z \times \Xi \times Y$ are bounded (namely, there exists a compact set $\mathcal{D}$ such that $(w(t), z(t), y(t), \eta(t)) \in \mathcal{D}$ for all $t \geq 0)$ and $\lim _{t \rightarrow \infty} y(t)=0$ uniformly in the initial conditions. Since the initial conditions of system (1) ranges in any arbitrarily large compact set, the problem is cast in a semiglobal framework, see [13]-[16].

In order to address this problem, we follow the so-called emulation approach. First we design the output regulator while ignoring the network. Then, we take the latter into account and a hybrid model is derived. Afterwards, we give conditions on the network, in terms of the MATI.

\footnotetext{
${ }^{1}$ In view of [16], we could also consider a more generic class of systems by considering $\dot{y}=q(w, z, y)+b(w, z, y) u$ where $b(w, z, y)>b>0$ for any $w, z, y \in \mathbb{R}^{r} \times \mathbb{R}^{n} \times \mathbb{R}$. However, to keep the notation as clear as possible, we will not address this class of nonlinear systems.

${ }^{2}$ See [14], [15] for a detailed definition of Poisson stability.
} 


\section{Solution In ThE CONTINUOUS-TIME CASE}

\section{A. Main assumptions and regulator design}

In this section we detail the structure of the regulator (3) solving the output regulation problem in the case in which the output $y$ is continuously measured. For this, we follow the framework proposed in [14]-[16]. We refer to these references for more details. We start by making the next assumption.

Assumption 1 There exists a function $\pi: \mathbb{R}^{r} \rightarrow \mathbb{R}^{n}$ smooth enough, a $C^{1}$ function $V_{0}$ and an open set $\mathcal{B} \subset \mathbb{R}^{r \times n}$ containing $W \times Z$ such that the following holds.

(i) The function $\pi$ satisfies $L_{s} \pi(w)=f(w, \pi(w), 0)$ for all $w \in W$.

(ii) The set $\mathcal{A}:=\left\{(w, z) \in W \times \mathbb{R}^{n}: z=\pi(w)\right\}$ is compact $^{3}$ and $\mathcal{A} \subset \mathcal{B}$.

(iii) There exist $\underline{\alpha}_{0}, \bar{\alpha}_{0} \in \mathcal{K}_{\infty}$ such that, for any $(w, z) \in \mathcal{B}$,

$$
\underline{\alpha}_{0}\left(|(w, z)|_{\mathcal{A} / \mathcal{B}}\right) \leq V_{0}(w, z) \leq \bar{\alpha}_{0}\left(|(w, z)|_{\mathcal{A} / \mathcal{B}}\right) .
$$

(iv) For any $c>0$, there exist $v>0$ such that, for any $(w, z)$ satisfying $V_{0}(w, z) \leq c$

$$
v|(w, z)|_{\mathcal{A} / \mathcal{B}}^{2} \leq V_{0}(w, z) .
$$

(v) For any $c, \bar{y}>0$ there exist $d, \beta>0$ such that, for any $(w, z)$ satisfying $V_{0}(w, z) \leq c$ and $|y| \leq \bar{y}$,

$\left\langle\nabla V_{0}(w, z), \operatorname{col}(s(w), f(w, z, 0))\right\rangle \leq-d V_{0}(w, z)+\beta|y|^{2}$.

Assumption 1 states that system (1) is (strongly) minimum-phase, namely the set $\mathcal{A}$ is exponentially stable for the zero-dynamics of (1) with a domain of attraction $\mathcal{B}$. Assumption 1 is stronger than the conditions in [15], where only asymptotic stability and local exponential stability of $\mathcal{A}$ are required in order to solve the problem of semiglobal output regulation. This is due to the fact that, similarly to [23], we need refine conditions in order to be able to compute explicit bounds of the parameters of the regulator proposed in [15]. Finally, the next extra assumption is stated, see [15].

Assumption 2 There exist an integer $d>0$ and a locally Lipschitz function $\varphi$ fulfilling

$$
L_{s}^{d} \sigma(w)=\varphi\left(\sigma(w), L_{s} \sigma(w), \ldots, L_{s}^{d-1} \sigma(w)\right)
$$

for all $w \in W$, where $\sigma(w):=-q(w, \pi(w), 0)$.

By following the design proposed in [15], the semiglobal output regulation problem can be solved in the case of continuous measurements by means of the following regulator

$$
\begin{aligned}
\dot{\xi} & =A \xi+B \varphi_{s}(\xi)-\kappa D_{g} K y \\
u & =C \xi-\kappa y
\end{aligned}
$$

where $\xi \in \mathbb{R}^{d}$ is the controller state with initial condition ranging in some compact set $\Xi \subset \mathbb{R}^{d},(A, B, C)$ is a triplet in prime form of dimension $d, K$ is a matrix to be chosen such that $A-K C$ is Hurwitz, $\varphi_{s}$ is any bounded

${ }^{3}$ See Assumption 1 in [15]. function that agrees on $W$ with the function $\varphi$ defined in Assumption $2, D_{g}$ is a diagonal matrix of dimension $d$ defined as $D_{g}=\operatorname{diag}\left(g, \ldots, g^{d}\right)$ and $g, \kappa \geq 1$ are positive high-gain parameters. It turns out that, if $g, \kappa$ are chosen large enough, namely $g \geq g^{\star}$ and $\kappa \geq \kappa^{\star}$ with $g^{\star}, \kappa^{\star} \geq 1$, output regulation is achieved according to [15, Proposition 1], and as shown below.

\section{B. Computation of the bounds}

In this section we provide explicit bounds for $g^{\star}, \kappa^{\star}$. Some steps are needed for this purpose. First, we will make a change of coordinates that allows to find an explicit Lyapunov function $V$ for the closed-loop system (1), (2), (4). Next, by using the Lipschitz constants of the nonlinear functions computed on a level set of $V_{0}$ containing the initial conditions, we explicitly provide bounds $g^{\star}, \kappa^{\star}$ via LMI conditions.

Let, for any $w \in W$,

$$
\Sigma(w):=\operatorname{col}\left(\sigma(w), L_{s} \sigma(w), \ldots, L_{s}^{d-1} \sigma(w)\right) .
$$

By Assumption 2, the function $\Sigma(\cdot)$ satisfies

$$
L_{s} \Sigma(w)=A \Sigma(w)+B \varphi(\Sigma(w))
$$

for any $w \in W$. Consider the following change of coordinates

$$
\xi \mapsto \eta:=D_{g}^{-1}(\xi-\Sigma(w))-K y .
$$

For the sake of convenience, we define $p:=\operatorname{col}(w, z)$ and, with some abuse of notation, we rewrite the $(w, z)$-system as $\dot{p}=f(p, y)$. The overall $(p, \eta, y)$-system reads as

$$
\begin{aligned}
& \dot{p}=f(p, y) \\
& \dot{\eta}=g(A-K C) \eta+B \Delta_{\varphi}+g L y-K \tilde{q}(p, y) \\
& \dot{y}=\tilde{q}(p, y)-(\kappa-g C K) y+g C \eta
\end{aligned}
$$

where $L:=A K-K C K$ and

$$
\begin{aligned}
\Delta_{\varphi}(p, \eta, y) & :=\frac{1}{g^{d-1}}\left[\varphi_{s}\left(\frac{D_{g}}{g} \eta+\Sigma(w)+K y\right)-\varphi(\Sigma(w))\right] \\
\tilde{q}(p, y) & :=q(w, z, y)+C \Sigma(w) \\
& =q(w, z, e)-q(w, \pi(w), 0) .
\end{aligned}
$$

Since the initial conditions of (1) and (4) range in the compact set $W \times Z \times \Xi \times Y$, the initial conditions of (6) also range in some given compact set $W \times Z \times E \times Y$ which, for $g \geq 1$, does not depend on $g$ itself. For (6), we define $V$ as, for $p \in \mathcal{B}, \eta \in \mathbb{R}^{d}, y \in \mathbb{R}$,

$$
V(p, \eta, y):=V_{0}(p)+\eta^{T} P \eta+\frac{1}{2} y^{2}
$$

where $V_{0}$ is the function given in Assumption 1 and $P=$ $P^{T}>0$ is computed as the solution of $P(A-K C)+(A-$ $K C)^{\top} P=-R$ for some $R=R^{T}>0$. Let $c>0$ be such that $\mathcal{V}=\{V(p, \eta, y) \leq c\}$ satisfies $\mathcal{V} \supset W \times Z \times E \times Y$. In view of item (i) of Assumption 1, such $c>0$ always exists since $V$ is radially unbounded. Let $\ell_{\varphi}, \ell_{q}>0$ be such that

$$
\begin{aligned}
\left|\Delta_{\varphi}(p, \eta, y)\right| & \leq \ell_{\varphi}(|\eta|+|K y|) \\
|\tilde{q}(p, y)| & \leq \ell_{q}\left(|p|_{\mathcal{A} / \mathcal{B}}+|y|\right)
\end{aligned}
$$

for all $(p, \eta, y) \in \mathcal{V}$. The existence of $\ell_{\varphi}, \ell_{q}$ is guaranteed by the fact that the set $\mathcal{V}$ is compact and the functions $\varphi, q$ 
are locally Lipschitz. Then, let $\beta$ in item (iii) of Assumption 1 be computed with $\bar{y}:=\sqrt{2 c}$. Finally, we define the matrix $S=S^{T}$ as follows

$$
S:=\left(\begin{array}{ccc}
v d & -s_{0} & -\frac{1}{2} s_{1} \\
\star & g \rho-s_{2} & -s_{3}-g s_{4} \\
\star & \star & \kappa-g C K-s_{5}
\end{array}\right)
$$

where $\rho$ denotes the minimum eigenvalue of $R, s_{0}:=$ $\ell_{q}|P K|, s_{1}:=\ell_{q}, s_{2}:=2 \ell_{\varphi}|P B|, s_{3}:=\ell_{\varphi}|P B||K|+$ $\ell_{q}|P K|, s_{4}:=\left(\frac{1}{2}+|L|\right), s_{5}:=\beta+\ell_{q}$. We have the next lemma, which follows from application of the Schur complement to $S$.

Lemma 1 There exists $g^{\star} \geq 1$ such that for any $g>g^{\star}$ there exists $\kappa^{\star} \geq 1$, such that, for any $\kappa>\kappa^{\star}$, the matrix $S$ defined in (9) satisfies $S>0$.

We are now ready to establish the following result stating that output regulation is achieved if $g, \kappa$ are taken as in Lemma 1. Though the bounds of $g$ and $\kappa$ are subject to some conservatism, this allows us to compute explicitly $g^{\star}, \kappa^{\star}$.

Theorem 1 Consider system (1), (2), (4) and suppose Assumptions 1 and 2 hold. Then, for any $g, \kappa$ satisfying $S>0$, output regulation is achieved, namely the trajectories of (1), (2), (4) are bounded for all $t \geq 0$ and $\lim _{t \rightarrow \infty} y(t)=0$ for all initial conditions in $W \times Z \times \Xi \times Y$.

Sketch of proof. By computing the derivative of the Lyapunov function (7) and by using standard Young's inequality, we obtain $\langle\nabla V, \tilde{f}(\chi)\rangle \leq-\psi^{T} S \psi$ for all $\chi \in \mathcal{V}$, where we used the compact notation $\dot{\chi}=\tilde{f}(\chi)$, with $\chi:=\operatorname{col}(p, \eta, y)$ and $\psi:=\operatorname{col}\left(|p|_{\mathcal{A} / \mathcal{B}},|\eta|,|y|\right)$. The proof concludes by using standard Lyapunov arguments and by noting that $\psi=0$ implies $y=0$.

\section{EMULATION-BASED SOLUTION}

\section{A. Hybrid model}

When the output $y$ is sampled, the regulator (4) becomes

$$
\begin{aligned}
\dot{\xi} & =A \xi+B \varphi_{s}(\xi)-\kappa D_{g} K \hat{y} \\
u & =C \xi-\kappa \hat{y} .
\end{aligned}
$$

By following [6], we introduce the sampling induced error $e$ defined as

$$
e:=y-\hat{y}
$$

In this work, we consider the simple case in which the sampled value $\hat{y}$ is kept constant during the transmission intervals, namely $\dot{\hat{y}}=0$ for $t \in\left(t_{i}, t_{i+1}\right), i \in \mathbb{Z}_{\geq 0}$, and it is reset to $y$ at transmission instants, namely $\hat{y}\left(t_{i}^{+}\right)=y\left(t_{i}\right)$ for any $i \in \mathbb{Z}_{\geq 0}$. In other words, a zero-order-hold device is used. As in [4], [5], [11], we introduce the clock $\tau$ to measure the time elapsed since the last transmission,

$$
\begin{array}{rllll}
\dot{\tau} & =1 & & \in[0, T] \\
\tau^{+} & =0 & & \in
\end{array}
$$

where $\tau \in \mathbb{R}$. By using the definition of $e$ and $\tau$, the system (1), (2) in closed-loop with the regulator (10) reads

$$
\left.\left.\begin{array}{rl}
\dot{w}=s(w) & \\
\dot{z} & =f(w, z, y) \\
\dot{\xi} & =A \xi+B \varphi_{s}(\xi)-D_{g} K \kappa(y-e) \\
\dot{y} & =q(w, z, y)+C \xi-\kappa(y-e) \\
\dot{e} & =q(w, z, y)+C \xi-\kappa(y-e) \\
\dot{\tau} & =1
\end{array}\right\} \begin{array}{l}
\tau \in[0, T] \\
w^{+}=w \\
z^{+}=z \\
\xi^{+}=\xi \\
y^{+}=y \\
e^{+}=0 \\
\tau^{+}=0
\end{array}\right\} \tau \in[v, T]
$$

with initial conditions ranging in the set $W \times Z \times \Xi \times Y \times$ $[-\delta, \delta] \times[0, T]$ for some $\delta>0$ which describe the maximum allowed initial error transmission. Clearly, if we suppose that a transmission occurs at the initial time, $e(0,0)=0$, and hence $e(0,0) \in[-\delta, \delta]$.

Our goal is to determine an explicit bound on $T$ so that the output regulation problem for system (12) is addressed, namely the solutions to (12) are complete, bounded and satisfy $\lim _{t+j \rightarrow \infty} y(t, j)=0$.

\section{B. Main Result}

Let $\gamma>0$ and

$$
Q:=\left(\begin{array}{cc}
S-\frac{1}{\gamma} H & -\kappa B_{3} \\
\star & \gamma
\end{array}\right)
$$

where $B_{3}=(0,0,1)^{T}$, and

$$
H:=\left(\begin{array}{ccc}
s_{1}^{2} & g s_{1} & s_{1}\left(\kappa-g C K+s_{1}\right) \\
\star & g^{2} & g\left(\kappa-g C K+s_{1}\right) \\
\star & \star & \left(\kappa-g C K+s_{1}\right)^{2}
\end{array}\right),
$$

where $S$ and $s_{1}$ are defined as in (9). We have the next result which follows by applying the Schur complement to $Q$.

Lemma 2 Let $g, \kappa$ be fixed according to Lemma 1 such that $S>0$. There exists $a \gamma^{\star}>0$ such that $Q>0$ for any $\gamma>\gamma^{\star}$, with $Q$ defined in (13).

We define the MATI as

$$
\mathcal{T}^{\star}(\kappa, \gamma):= \begin{cases}\frac{1}{\kappa r} \arctan (r) & \gamma>\kappa \\ \frac{1}{\kappa} & \gamma=\kappa \\ \frac{1}{\kappa r} \operatorname{arctanh}(r) & \gamma<\kappa,\end{cases}
$$

where

$$
r=\sqrt{\left|\left(\frac{\gamma}{\kappa}\right)^{2}-1\right|}
$$

The next theorem states that, if the parameters $T, \kappa, g$ are properly chosen, the trajectories of the closed-loop system (12) are bounded and the regulated output $y$ converges 
asymptotically to zero, namely the problem of semiglobal output regulation under sampling measurements is solved.

Theorem 2 Suppose that Assumptions 1, 2 hold and consider system (12) with $g, \kappa \geq 1$ fixed according to Theorem 1 and let $\gamma>0$ be chosen such that the matrix $Q$ defined in (13) satisfies $Q>0$. There exists $\delta>0$ such that, for any $T \in\left(0, \mathcal{T}^{\star}(\kappa, \gamma)\right)$, with $\mathcal{T}^{\star}(\kappa, \gamma)$ defined in (15), the solutions to (12), starting in $W \times Z \times \Xi \times Y \times[-\delta, \delta] \times[0, T]$, are complete, bounded and satisfy $\lim _{t+j \rightarrow \infty} y(t, j)=0$.

Sketch of proof. Let $g, \kappa, \gamma>0$ be fixed according to Theorem 1 and Lemma 2 so that $Q>0$. By using the function $V$ in (7), let $b:=\sup _{(p, \eta, y) \in W \times Z \times E \times Y} V(p, \eta, y)$. By construction, $b<c$, with $c$ defined after (7). Let $t_{\max }=$ $\mathcal{T}^{\star}(\kappa, \gamma)$. In view of the results in [5], [6, Claim 1], there exist $\lambda \in(0,1)$ and a function $\phi:\left[0, t_{\max }\right] \rightarrow \mathbb{R}$ solution to

$$
\dot{\phi}=-2 \kappa \phi-\gamma\left(\phi^{2}+1\right), \quad \phi(0)=\lambda^{-1}
$$

so that $\phi(t) \in\left[\lambda, \lambda^{-1}\right]$ for all $t \in\left[0, t_{\max }\right]$. Select $\delta=$ $\sqrt{\lambda(c-b)}$. Finally, fix any $T \in\left(0, \mathcal{T}^{\star}(\kappa, \gamma)\right)$.

Let $x=\operatorname{col}(p, \eta, y, e, \tau)$. We write system (12) as

$$
\dot{x}=F(x) \quad \text { if } \quad x \in \mathcal{C}, \quad x^{+}=G(x), \quad \text { if } \quad x \in \mathcal{D},
$$

where $\mathcal{C}:=\mathcal{B} \times \mathbb{R}^{d} \times \mathbb{R} \times \mathbb{R} \times[0, T]$ and $\mathcal{D}:=\mathcal{B} \times \mathbb{R}^{d} \times$ $\mathbb{R} \times \mathbb{R} \times[v, T]$. For the sake of compactness, we denote the set of initial conditions of (16) as $X:=W \times Z \times E \times Y \times$ $[-\delta, \delta] \times[0, T]$. The rest of the proofs follows by applying the same type of arguments as in [5], [6]. with the Lyapunov function function $U(x):=V(p, \eta, y)+\phi(\tau)|e|^{2}$ where $V$ is the function defined in (7). In particular, we have $\mathcal{U}:=\{x$ : $U(x)<c\} \supset X$ and $\varrho_{0}(\omega(x)) \leq U(x) \leq \varrho_{1}(\omega(x))$ for some $\varrho_{0}, \varrho_{1} \in \mathcal{K}_{\infty}$ and for all $x \in(\mathcal{C} \cup \mathcal{D} \cup G(\mathcal{D})) \cap \mathcal{U}$, where

$$
\omega(x):=|p|_{\mathcal{A} / \mathcal{B}}+|\eta|+|y|+|e|+|\tau|_{[0, T]} .
$$

The function $\omega$ is a proper indicator for the compact set

$$
\mathcal{I}:=\mathcal{A} \times\{0\} \times\{0\} \times\{0\} \times[0, T]
$$

on $(\mathcal{C} \cup \mathcal{D} \cup G(\mathcal{D})) \cap \mathcal{U}$. By evaluating $U$ at jumps we have $U(G(x)) \leq U(x)$ for all $x \in \mathcal{U}$. Similarly, during flows we obtain (by applying standard Young's inequalities) $\langle\nabla U(x), F(x)\rangle \leq-\psi_{e}^{T} Q \psi_{e}$ for all $x \in \mathcal{U}$, where we used the compact notation $\dot{x}=F(x), \psi_{e}:=\operatorname{col}(\psi,|e|)$, with $\psi$ defined in the proof of Theorem 1 . The latter inequality implies $\langle\nabla U(x), F(x)\rangle \leq-\tilde{\rho}(U(x))$ for some function $\tilde{\rho}$, quadratic around the origin. As a consequence by applying of [24, Proposition 3.27], we conclude that the set $\mathcal{I}$ is preasymptotically stable on $\mathcal{U}$. In view of the definition of the $\tau$-dynamics, system (16) satisfies the viability condition [24, Proposition 6.10], and solutions cannot jump out of $\mathcal{C} \cup \mathcal{D}$. Moreover, since the set $\mathcal{I}$ is compact and pre-asymptotically stable with a domain of attraction containing $X$, solutions to (16) starting in $X$ cannot have finite escape time. As a result, according to [24, Proposition 6.10], solutions are complete (recall that we call solutions, maximal solutions). In addition, we have the guarantee that solutions to (16) starting in $X$ are bounded. This shows boundedness of solutions to (12) in view of (5). Finally, because of the asymptotic stability of the set $\mathcal{I}$ and the completeness of solutions, we have $\lim _{t+j \rightarrow \infty} \omega(x(t, j))=0$. By definition of $\omega$, this implies $\lim _{t+j \rightarrow \infty} y(t, j)=0$, concluding the proof.

Theorem 2 states that output regulation can be achieved in case of sampled measurements if the transmission intervals are small enough. Note that the domain of attraction cannot contain arbitrarily large initial error transmissions $e(0,0)$ as must lie in the bound $[-\delta, \delta]$. As previously remarked, if a transmission occurs at the initial time, this condition holds for free. The bound in (15) depends on the parameters $\kappa$ and $\gamma$ and thus on $g$, since so does $\kappa$. In view of this expression, we obtain that, in general, the larger $\kappa$, the smaller $\mathcal{T}^{\star}$, though transient effect may occur, as shown on an example in Section V, see Figure 2.

Remark. Since the MATI depends directly on the parameters of the controller (4), one could ask if it is possible to compute the controller gains $g, \kappa$ that give the largest MATI. However, this issue, which is somehow dual to the problem investigated in this paper, is very difficult, since we would need tighter bounds on $g$ and $\kappa$ in the continuous time case. This is in general not possible since the stability analysis of the system (6) is too coarse, and therefore the bounds provided in Theorem 1 are often subject to some conservatism. This is confirmed by the fact that, to the best of authors knowledge, no explicit bounds for $g, \kappa$ are given in literature and a finer analysis is unknown in the considered framework. Nevertheless, the bounds on $S$ and $Q$ could be probably refined by considering the vector states $z, \eta$ and not their norms.

\section{EXAMPLE}

We consider system (1) with

$$
f(w, z, y)=-2 z+\alpha(y), \quad q(w, z, y)=2 y-2 z-w_{1} .
$$

where $\alpha(\cdot)$ is any globally Lipschitz functions satisfying $|\alpha(y)|^{2} \leq|y|^{2}$. We suppose that exosystem (2) is generated by the following nonlinear oscillator (see [25])

$$
s\left(w_{1}, w_{2}\right)=\left(\begin{array}{c}
-w_{1}+\arctan \left(\beta w_{1}-w_{2}\right) \\
\epsilon\left(w_{1}-w_{2}\right)
\end{array}\right)
$$

where $\beta>1$ and $\epsilon \in(0,1)$. In the simulations, we have chosen $\beta=1.2$ and $\epsilon=0.1$. It can be verified by simulations that with such values, system (2) admits a limit cycle contained in a compact set $W$ satisfying $\sup _{w \in W}|w| \leq 0.6$. Assumption 1 holds with $V_{0}(z)=\frac{1}{2} z^{2}, v=\frac{1}{2}, d=1, \beta=1$, $\ell_{q}=2$. Concerning Assumption 2, it is verified with $d=2$ and $\varepsilon$ given by

$$
\varphi(\xi)=-\xi_{2}+\frac{\epsilon(\beta-1) \xi_{1}+\beta \xi_{2}-\epsilon \tan \left(\xi_{1}+\xi_{2}\right)}{1+\tan \left(\xi_{1}+\xi_{2}\right)^{2}} .
$$

Function $\varphi$ is globally Lipschitz with Lipschitz constant $\ell_{\varphi}=1+\beta=2.2$. Finally, we take $K, P$ as $K=\operatorname{col}(3,2)$ and $P=\left(\begin{array}{cc}0.5 & -0.5 \\ -0.5 & 1\end{array}\right)$. As a result, the elements of $S$ in (9) are $s_{0}=\sqrt{2}, s_{1}=2, s_{2}=4.9193, s_{3}=10.2827$, 
$s_{4}=9.7195, s_{5}=3.2$. We select different values of $g, \kappa$ satisfying $S>0$ to investigate their impact on the MATI bound, and we select the minimum value of $\gamma$, denoted as $\gamma^{\star}$, such that the matrix $Q$ defined in (13) satisfies $Q>0$. Finally, we compute $\mathcal{T}^{\star}\left(\kappa, \gamma^{\star}\right)$ using (15).

Figure 2 shows the dependency of $\mathcal{T}^{\star}$ and $\gamma^{\star}$ on $\kappa$ for a fixed $g$, exhibiting a "parabola" effect and suggesting that an optimal tuning of the high-gain parameters $g, \kappa$ should be followed in order to maximize the MATI. We compared those values with the maximum allowed time interval $T_{\text {simu }}$ found by simulating the closed-loop system with a fixed sampling interval. The values are reported in Table I. We can see that the ratio between the MATI $T_{\text {sim }}$ computed by simulations and the MATI $\mathcal{T}^{*}\left(\kappa, \gamma^{\star}\right)$ is always of the order of 10 . This suggests that the analysis in Section IV-B provides a good approximation of the MATI, when the parameters $g, \kappa, \gamma$ are chosen according to Lemmas 1 and 2.
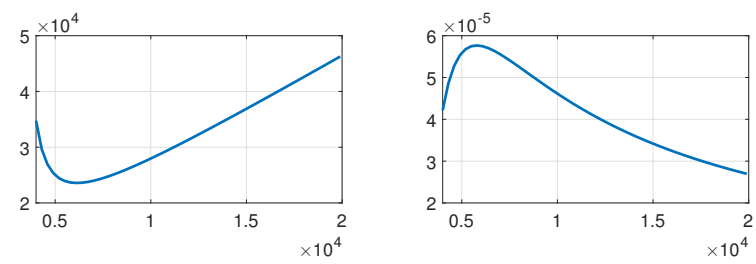

Fig. 2. Figure a: plot of $\gamma^{\star}$ as a function of $\kappa$ for a fixed $g$. Figure b: plot of $\mathcal{T}^{\star}\left(\kappa, \gamma^{\star}\right)$ as a function of $\kappa$ and $\gamma^{\star}(\kappa)$, for a fixed $g$.

\begin{tabular}{|c|c|c|c|c|c|c|}
\hline$g$ & 19.2 & 19.2 & 19.2 & 40 & 40 & 40 \\
\hline$\kappa\left(\cdot 10^{3}\right)$ & 3.98 & 4.2 & 5 & 5.34 & 5.8 & 6.2 \\
\hline$\gamma^{\star}\left(\cdot 10^{4}\right)$ & 3.53 & 3.09 & 2.5 & 9.37 & 6 & 4.9 \\
\hline $\mathcal{T}^{*}\left(\cdot 10^{-5}\right)$ & 4.15 & 4.68 & 5.59 & 1.62 & 2.46 & 2.95 \\
\hline$T_{\operatorname{sim}}\left(\cdot 10^{-4}\right)$ & 5 & 4 & 3.5 & 3.5 & 2 & 3 \\
\hline$T_{\operatorname{sim}} / \mathcal{T}^{*}$ & 12 & 8.5 & 6.62 & 21.6 & 8.1 & 10.1 \\
\hline
\end{tabular}

TABLE I

Values of $\mathcal{T}^{\star}(\kappa, \gamma)$ in (15) and $T_{\text {sim }}$ for some values of $g, \kappa, \gamma^{\star}$.

\section{CONCLUSION}

We studied the properties of nonlinear internal model regulators for minimum-phase systems with sampled measurements. We showed that if the sampling time is small enough, the classical design introduced in [15] guarantees that the tracking error converges to zero, despite the sampling. An explicit formula for the maximum allowed time interval (MATI) is provided. Optimal choice of the values of $g, \kappa$ to maximize the value of the MATI is a future research direction, as suggested by the interpretation of Figure 2. Another natural extension of this work is to consider the case in which also the input is sampled. In this case, we expect that asymptotic output regulation may not be achieved in general, while practical regulation could be ensured. This problem is not a trivial extension of the sampled-output case and it is currently under investigation.

\section{REFERENCES}

[1] J. P. Hespanha, P. Naghshtabrizi and Y. Xu, "A Survey of Recent Results in Networked Control Systems", Proceeding of IEEE, 95(1), pp. 138162,2007
[2] T. C. Yang, "Networked control system: a brief survey", IEEE Proceedings-Control Theory and Applications, 153(4), pp. 403-412, 2006.

[3] W. Heemels, A. Teel, N. van de Wouw, and D. Nešić, "Networked control systems with communication constraints: tradeoffs between transmission intervals, delays and performance", IEEE Transactions on Automatic Control, vol. 55 (8), pp. 1781-1796, 2010.

[4] D. Nešić and D. Liberzon, "A unified framework for design and analysis of networked and quantized control systems", IEEE Transactions on Automatic Control, vol. 54, pp. 732-747, 2009.

[5] D. Carnevale, A. Teel, and D. Nešić, "A Lyapunov proof of an improved maximum allowable transfer interval for networked control systems", IEEE Transactions on Automatic Control, vol. 52 (5), pp. 892-897, 2007.

[6] D. Nešić, A. Teel, D. Carnevale, "Explicit computation of the sampling period in emulation of controllers for nonlinear sampled-data systems", IEEE Transactions on Automatic Control, vol. 54(3), pp. 619-624, 2009.

[7] R. Postoyan and D. Nešić, "A framework for the observer design for networked control systems", IEEE Transactions on Automatic Control, vol. 57(5), pp. 1309-1314, 2012.

[8] F. Ferrante, F. Gouaisbaut, , R. G. Sanfelice, S. Tarbouriech, "State estimation of linear systems in the presence of sporadic measurements", Automatica, vol. 73, pp. 101-109, 2016

[9] H. Gao and T. Chen, "Network-based $H_{\infty}$ output tracking control", IEEE Transactions on Automatic Control, vol. 53 (3), pp. 655-667, 2008.

[10] N. van de Wouw, P. Naghshtabrizi, M. Cloosterman, and J. Hespanha, "Tracking control for sampled-data systems with uncertain sampling intervals and delays", Int. J. of Robust and Nonlinear Control, vol. 20 (4), pp. 387-411, 2010.

[11] R. Postoyan, N. van de Wouw, D. Nešić and W.P.M.H. Heemels, "Tracking control for nonlinear networked control systems", IEEE Transactions on Automatic Control, vol. 59(6), pp. 1539-1554, 2014.

[12] B. A. Francis, and W. M. Wonham. "The internal model principle of control theory", Automatica, vol. 12, pp. 457-465, 1976.

[13] A. Serrani, A. Isidori and L. Marconi, "Semiglobal robust output regulation of minimum-phase nonlinear systems", Int. Journal of Robust Nonlinear Control, vol. 10, pp. 379-396, 2000.

[14] C.I. Byrnes and A. Isidori, "Limit Sets, Zero Dynamics, and Internal Models in the Problem of Nonlinear Output Regulation", IEEE Transactions on Automatic Control, vol. 48(10), pp. 1712-1723, 2003.

[15] C.I. Byrnes and A. Isidori, "Nonlinear Internal Models for Output Regulation", IEEE Transactions on Automatic Control, vol. 49(12), pp. 2244-2247, 2004.

[16] D. Astolfi, A. Isidori and L. Marconi, "Output regulation via lowpower construction", in Feedback stabilization of controlled dynamical systems. N. Petit (ed). Springer, 2017.

[17] L. Marconi, L. Praly and A. Isidori, "Output stabilization via nonlinear Luenberger observers", SIAM. J. Control Optim., vol 45 (6), pp. 22772298, 2007.

[18] A. Isidori, L. Marconi and G. Casadei, "Robust output synchronization of a network of heterogeneous nonlinear agents via nonlinear regulation theory", IEEE Transactions on Automatic Control, vol. 59(10), pp. 26802691, 2014.

[19] D.A. Lawrence. and E. A. Medina, "Output Regulation for Linear Systems with Sampled Measurements", American Control Conference, pp. 2044-2050, 2001.

[20] B. Castillo, S. Di Gennaro, S. Monaco, and D. Normand-Cyrot, "On Regulation Under Sampling", IEEE Transactions on Automatic Control, vol. 42(6), pp. 864-868, 1997.

[21] O. M. Grasselli, S. Longhi, A. Tornambe, and P. Valigi, "Robust ripple-free regulation and tracking for parameter-dependent sampled-data systems, IEEE Transactions on Automatic Control, vol. 41(7), pp. 1031$1037,1996$.

[22] A. Isidori, L. Marconi and C. De Persis, "Remote Tracking via Encoded Information for Nonlinear Systems", Systems \& Control Letters, vol. 55, pp. 809-818, 2006.

[23] W. Liu and J. Huang, "Event-Triggered Global Robust Output Regulation for a Class of Nonlinear Systems", IEEE Transactions on Automatic Control, vol. 62 (11), pp. 5923-5930, 2017.

[24] R. Goebel, R. Sanfelice and A. Teel, Hybrid Dynamical Systems: Modeling, Stability, and Robustness. Princeton University Press, 2012.

[25] Y. Tang, A. Franci, R. Postoyan, "Qualitative parameter estimation for a class of relaxation oscillators", IFAC World Congress, pp. 2929-2934, Toulouse, France, 2017. 